\title{
Decision-making deficit in chronic migraine patients with medication overuse
}

\author{
B. Biagianti • L. Grazzi • O. Gambini · \\ S. Usai $\cdot$ R. Muffatti $\cdot$ S. Scarone $\cdot$ G. Bussone
}

(C) Springer-Verlag 2012

\begin{abstract}
Patients with chronic migraine developing medication-overuse headache (MOH) show dependencylike behaviors such as loss of control over analgesics despite adverse consequences on headaches, high rates of relapse after withdrawal from symptomatic medications, and compromised social functioning. Neuroimaging research suggests a common pathophysiology between substance-use disorders and $\mathrm{MOH}$, which involves functional alterations in fronto-striatal networks, particularly in the orbitofrontal region of prefrontal cortex. These findings could explain the impaired decision-making observed in substance-use disorders. We hypothesize that $\mathrm{MOH}$ could share fronto-striatal circuit dysfunction and relative decision-making deficit with addiction. We further examine whether this deficit is a persistent cognitive trait or a reversible consequence of medication overuse. This study shows a dataset of 50 patients with $\mathrm{MOH}$ before the detoxification. All patients underwent a complete neurological and psychiatric examination. Psychiatric examination consisted of a clinical interview, Structured Clinical Interview for DSM-IV TR Axis II Personality Disorders, Anxiety and Depression Hamilton Scales, Severity of Dependence Scale. The neurological examination included the migraine disability assessment questionnaire.
\end{abstract}

\footnotetext{
B. Biagianti $(\bowtie) \cdot$ O. Gambini $\cdot$ S. Scarone

Department of Psychiatry, University of Milan Medical School and San Paolo Hospital, via A. di Rudinì 8/A, 20142 Milan, Italy e-mail: bruno.biagianti@gmail.com

L. Grazzi · S. Usai · G. Bussone

Headache Center, National Neurological Institute C. Besta,

Milan, Italy

R. Muffatti

Department of Psychiatry, San Paolo Hospital, Milan, Italy
}

Neuropsychological assessment of fronto-striatal circuits was investigated using the Iowa gambling task (IGT). Twenty patients monitored for any relapse into medication overuse had 12 months of follow-up. Our sample, characterized by high rates of disability and dependency-like behaviors, exhibited a deficit in IGT performance, indicating an overall impairment in decision-making. All the 20 patients showed neurological and psychiatric improvement at 12-month follow-up, notwithstanding the overuse relapse, but a persistent IGT deficit was found. To our knowledge this is the first study that assesses this cognitive function in patients with $\mathrm{MOH}$. Medication-overuse headache seems to share a persistent decision-making deficit with substance abuse that confirms the orbitofrontal cortex hypometabolism described in literature from a neuropsychological perspective. Looking at these shared neurocognitive features, our results suggest that $\mathrm{MOH}$ could belong to the addiction spectrum. Fronto-striatal dysfunction could be a premorbid psychobiological condition of vulnerability explaining the clinical onset of medication overuse and recurrent relapses. We propose that IGT could be used to identify chronic migraine patients with higher risk for medication overuse and relapse.

Keywords Orbitofrontal cortex - Iowa gambling task · Decision-making - Medication-overuse headache .

Addiction

\begin{tabular}{|c|c|}
\hline \multicolumn{2}{|c|}{ Abbreviations } \\
\hline $\mathrm{MOH}$ & Medication-overuse headache \\
\hline fMRI & Functional magnetic resonance imaging \\
\hline $\mathrm{OFC}$ & Orbitofrontal cortex \\
\hline PET & Positron emission tomography \\
\hline ST & Iowa gambling task \\
\hline
\end{tabular}




\section{Introduction}

From 30 to $50 \%$ of chronic migraineurs who, during the natural history of their disease, experience an increase of episode frequency and intensity, start overusing symptomatic drugs for migraine in order to cope with pain and disability. They enter a vicious cycle where overuse of medications no longer controls headaches and becomes responsible of the onset of a secondary headache with variable clinical features, called medication-overuse headache $(\mathrm{MOH})[1,2]$.

Beyond the necessity of coping with the increased pain and disability, some dependency-like behaviors are being pointed out as possible reasons for medication overuse [3-5]. Loss of control over the use of painkillers despite the adverse consequences on headaches (unmanageability) and high rates of relapse (powerlessness) occur in a high proportion of patients (30-40\% at 1 year, $40-70 \%$ after 4-5 years) [2]. Pharmacological tolerance, cephalalgiaphobia, high rates of disability and compromised social functioning are often observed in these patients. Taken together, these findings brought many authors to consider MOH a bio-behavioral disorder [3-6]. Both the Fuh and Radat studies found around $70 \%$ of comorbidity between $\mathrm{MOH}$ and substance abuse according to DSM-IVTR $[4,7]$.

Chronic exposure to addictive drugs is shown to be associated with impairment of several neural networks, including the fronto-striatal circuit, which involves the prefrontal regions (including the dorsolateral cingulate anterior and orbitofrontal areas), the limbic system (amygdala, entorhinal cortex), and their projections to the ventral striatum $[8,9]$.

The impairment of this circuit results in prefrontal cortex impairment with a relative dominance of limbic regions on behavior. As prefrontal regions no longer guide the behavior toward controlled, rational and appropriate choices, limbic dominance causes a loss of control, and an increase of impulsivity and semi-automatic actions [10].

In the meta-analysis on impaired decision-making in addicts by Dom et al. [11], ten samples of patients with various substance-use disorders exhibited impairment in decision-making tasks relative to controls.

Some functional neuroimaging studies have investigated the relationship between decision-making deficit and neural activation in different prefrontal areas. Both the fMRI study by Paulus et al. [12] on meta-amphetamine addicts, and the PET study by Bolla et al. [13] on cocaine abusers, gave evidence that the decision-making deficit shown by these samples compared to controls was consistent with both orbitofrontal and dorsolateral prefrontal dysfunction.

A commonality between $\mathrm{MOH}$ and addiction arose from a PET study by Fumal et al. [14], who found a persistent hypometabolism of OFC 3 weeks before and 3 weeks after the detoxification in $\mathrm{MOH}$ patients, suggesting that this dysfunction might predispose certain migraineurs to $\mathrm{MOH}$ and to relapse after the detoxification.

To our knowledge, while various studies have focused on neuropsychology among those with substance-use disorders, this is the first attempt to evaluate decision-making in $\mathrm{MOH}$ patients through a neuropsychological task, the Iowa gambling task (IGT).

Designed to assess patients with ventromedial lesions of the prefrontal cortex, this computer-based card task is known to evaluate the decision-making function [15].

The task consists of picking cards from four decks, each of which can give unpredictable rewards or penalties. In order to maximize an initial $€ 2,000$ loan of play money, participants need to weigh short-term rewards against longterm losses. In fact, the two risky decks (A and B) give high rewards $(€ 100)$, but total penalties outweigh them. Otherwise, decks $\mathrm{C}$ and $\mathrm{D}$ give small but safer rewards $(50 €)$. The optimal strategy is to integrate the value of these varying rewards and penalties over time, avoiding the short-term appeal of decks A and B in favor of the slower gain from decks $\mathrm{C}$ and $\mathrm{D}$. Performance on the gambling task is evaluated through a net score, which corresponds to the number of cards drawn from the advantageous decks minus the number of cards drawn from the disadvantageous ones. When negative, the net score expresses indeed a decision-making deficit.

Wondering as to whether or not $\mathrm{MOH}$ patients would show the same decision-making impairment previously observed in addicts [11], we decided to compare the IGT performances of $\mathrm{MOH}$ patients and healthy gender, and age-matched controls. Furthermore, we investigated the nature of this possible deficit, whether this was a temporary consequence of medication overuse or a persistent feature.

\section{Materials and methods}

A sample of 50 patients, from 18 to 65 years of age with a history of chronic migraine (i.e., present at least 15 days a month on a regular basis for at least 3 months) and medication overuse (according to the latest ICHD-II criteria, category 8.2.7) [16], were enrolled in the Headache Clinic of the Neurological Institute Carlo Besta in Milan.

The first psychiatric evaluation included: Structured Clinical Interview for DSM-IV TR Axis II Personality Disorders, Hamilton Rating Scales for Anxiety and Depression, migraine disability assessment (MIDAS) questionnaire and severity of dependence scale (SDS).

The MIDAS questionnaire was developed to measure the headache-related disability. It attempts to determine how many days were affected in a patient's life to the point that he/she was unable to function in a usual way. Migraine 
disability assessment takes into account the last 3 months when asking the questions $[17,18]$. The SDS is a five-item questionnaire, originally created to investigate opiate dependence, recently used in people with primary and secondary chronic headache to detect patterns of medication overuse and dependency-like behaviors [19, 20]. In order to assess decision-making, both patients and healthy age- and gender-matched controls underwent the Italian version of the computer-based IGT [15].

The demographic, clinical, and psychopathological characteristics of the patient population are described as means and standard deviations, and reported in Table 1. Twelve months later long-term monitoring was set up with the same battery of tests in an outpatient regimen in order to detect any relapse into medication overuse and dependence. In the follow-up data elaboration, $t$ test and Chi-square were, respectively, used for comparison of continuous or parametric variables (Mann-Whitney and Fisher exact test, as appropriate). Type I and II errors were possible because of the number of subjects and comparisons. In consideration of the exploratory nature of the study, we referred to levels of significance of $P \leq 0.05$, without operating any correction for multiple comparisons.

\section{Results}

Of the $50 \mathrm{MOH}$ patients included in the study, most patients had continuous headache with superimposed episodes of acute headache (mean frequency of episodes per month was 21.96 with $6.59 \mathrm{SD}$ ) and daily drug intake (mean number of tablets per month was 31.04 with 22.51 SD). The overused medications were triptans alone (20 patients, $40 \%$ ), simple analgesics alone or in combination with caffeine (26 patients, $52 \%$ ) and triptans plus analgesics (six patients, $12 \%$ ).

Twenty-two patients (44\%) and 14 patients (28\%), respectively, had a past and present Axis 1 psychiatric diagnosis (anxiety disorder, major depressive disorder). None fulfilled DSM-IVTR criteria for personality disorders.

The sample was characterized by high rates of disability (mean MIDAS score was 69.60 with 49.18 SD) and severe dependency-like behaviors due to anti-migraine drugs (mean SDS score was 8.73 with $2.23 \mathrm{SD}$ ): 47 patients (94\%) achieved an SDS score greater than or equal to 5, the cut off for diagnosing the behavioral addiction for painkillers [20].

Our sample of $\mathrm{MOH}$ patients showed a statistically significant deficit in decision-making (mean net score on IGT was -10.40 with $15.82 \mathrm{SD}$ ) compared to healthy gender- and age-matched controls (independent sample $t$ test with $t$ 8.08; $d f$ 79.6, sig two-tailed 0.000).

The dataset for the longitudinal study is now available for the 20 patients: according to ICHD-II criteria [16], 13 of them $(65 \%)$ discontinued medication overuse after the detoxification and restored an episodic migraine pattern; 7 patients $(35 \%)$ relapsed into $\mathrm{MOH}$ at 12-month follow-up, despite the temporary resolution of medication overuse soon after the detoxification.

Observing the clinical and psychopathological score transition in the follow-up sample regardless of relapse (Table 2), we found very significant decreases in MIDAS score, pain intensity, frequency of episodes, and number of tablets per month. In contrast to this global improvement, the mean net score in IGT performance remained negative (-7.70 with 16.92 SD), with a 1.00 statistical significance in the paired samples $t$ test, showing a persistent deficit in decision-making.
Table 1 Demographic and clinical features of medicationoveruse headache $(\mathrm{MOH})$

\begin{tabular}{lccrr}
\hline & \multicolumn{3}{l}{ Baseline during withdrawal $(N=50)$} & \\
\cline { 2 - 5 } & Minimum & Maximum & Mean & SD \\
\hline AGE (years) & 23.00 & 65.00 & 41.28 & 9.96 \\
Migraine duration (years) & 1.00 & 60.00 & 22.13 & 13.05 \\
Medication overuse duration (years) & 0.25 & 36.00 & 2.99 & 6.57 \\
Frequency of episodes per month & 15.00 & 30.00 & 21.96 & 6.59 \\
Number of tablets per month & 14.00 & 90.00 & 31.04 & 22.51 \\
Pain intensity on VAS & 3.00 & 10.00 & 7.80 & 1.64 \\
HAM-A score & 0.00 & 30.00 & 13.19 & 7.91 \\
HAM-D score & 0.00 & 39.00 & 12.60 & 7.56 \\
Iowa gambling task net score & -52.00 & 14.00 & -10.40 & 15.82 \\
Severity of dependence scale & 4.00 & 13.00 & 8.73 & 2.23 \\
MIDAS total score & 7.00 & 270.00 & 69.60 & 49.18 \\
\hline
\end{tabular}


Table 2 Follow-up dataset for continuous variables (paired samples statistics and $t$ test)

\begin{tabular}{|c|c|c|c|c|c|c|c|}
\hline & \multicolumn{3}{|c|}{ Baseline during withdrawal $(N=20)$} & \multicolumn{3}{|c|}{1 -year follow-up $(N=20)$} & \multirow{2}{*}{$\begin{array}{l}t \text { test } \\
\text { Sig (two-tailed) }\end{array}$} \\
\hline & Mean & SD & SE mean & Mean & SD & SE mean & \\
\hline Frequency of attacks per month & 22.15 & 6.42 & 1.44 & 11.60 & 7.32 & 1.64 & 0.00 \\
\hline Number of tablets per month & 30.65 & 22.14 & 4.95 & 16.50 & 14.77 & 3.30 & 0.03 \\
\hline Pain intensity on VAS & 7.85 & 1.46 & 0.33 & 6.60 & 1.76 & 0.39 & 0.01 \\
\hline HAM-A score & 12.30 & 6.60 & 1.48 & 11.60 & 6.22 & 1.39 & 0.67 \\
\hline HAM-D score & 12.00 & 6.52 & 1.46 & 9.10 & 5.46 & 1.22 & 0.03 \\
\hline Iowa gambling task net score & -7.70 & 17.32 & 3.87 & -7.70 & 16.92 & 3.78 & 1.00 \\
\hline MIDAS total score & 65.05 & 37.08 & 8.29 & 33.25 & 32.65 & 7.30 & 0.00 \\
\hline
\end{tabular}

\section{Discussion}

The aim of this study was to show that migraineurs overusing painkillers, although forced to cope with chronic pain, often develop a behavioral addiction that might rise from the same decision-making deficit often described in substance abuse [11]. Moreover, follow-up at 12 months showed that, contrary to the global clinical and psychopathological improvement observed in these patients, decision-making was persistently impaired. Still, our data confirm the OFC hypometabolism described by Fumal et al. [14] in his PET study from a neuropsychological perspective. Taken together, this data show, in a preliminary but promising way, that even a non-psychotropic drug dependence can be associated with the fronto-striatal circuit impairment and decision-making deficit traditionally observed in different populations of addicts $[8,9,11]$, and more recently also in people with binge eating disorder and pathological gambling. Thus, there is growing evidence in considering $\mathrm{MOH}$ part of the addiction spectrum [21].

After attesting to a persistent decision-making deficit in patients with $\mathrm{MOH}$, yet recognizing the current small size of our follow-up sample, further studies are needed to elucidate the role of fronto-striatal circuit dysfunction. We believe that the impairment of fronto-striatal networks might constitute a psychobiological vulnerability [9] that, in this particular condition, influences the clinical onset of medication overuse and recurrent relapses in some patients with chronic migraine. Therefore, clinicians will hopefully use the IGT as a valid tool to identify chronic migraine patients at a higher risk for medication overuse and relapse, who might potentially require stricter monitoring.

Conflict of interest No current conflicts of interest for any of the authors.

\section{References}

1. Diener HC, Katsarava Z, Limmroth V (2010) Headache attributed to a substance or its withdrawal. Handb Clin Neurol 97:589-599
2. Katsarava $\mathrm{Z}$ et al (2010) Medication overuse headache: rates and predictors for relapse in a 4 -year prospective study. Cephalalgia 25(1):12-15

3. Calabresi $\mathrm{P}$ et al (2005) MOH: similarities with drug addiction. Trends Pharmacol Sci 26(2):62-68

4. Radat $\mathrm{F}$ et al (2010) What is the role of dependence-related behavior in medication-overuse headache? Headache 50(10): 1597-1611

5. Andrasik F, Grazzi L, Usai S, D’Amico D, Kass S, Bussone G (2007) Disability in chronic migraine with medication overuse: treatment effects at 3 years. Headache 47(9):1277-1281

6. Saper JR, Hamel RL, Lake AE (2005) Medication overuse headache is a bio-behavioral disorder. Cephalalgia 25:545-546

7. Fuh JL et al (2010) Does medication overuse headache represent a behavior of dependence? Pain 119(1-3):49-55

8. Volkow ND et al (2004) The addicted human brain viewed in the light of imaging studies: brain circuits and treatment strategies. Neuropharmacology 47(Suppl 1):3-13

9. Schoenbaum $\mathrm{G}$ et al (2008) The role of orbitofrontal cortex in drug addiction: a review of preclinical studies. Biol Psychiatry 63(3):256-262

10. Crews FT, Boettiger CA (2009) Impulsivity, frontal lobes and risk for addiction. Pharmacol Biochem Behav 93(3):237-247

11. Dom G et al (2005) Substance use disorders and the orbitofrontal cortex: systematic review of behavioral decision-making and neuroimaging studies. Br J Psychiatry 187:209-220

12. Paulus MP et al (2003) Decision making by methamphetaminedependent subjects is associated with error-rate-independent decrease in prefrontal and parietal activation. Biol Psychiatry 53(1):65-74

13. Bolla KI et al (2003) Orbitofrontal cortex dysfunction in abstinent cocaine abusers performing a decision-making task. Neuroimage 19(3):1085-1094

14. Fumal A et al (2006) Orbitofrontal cortex involvement in chronic analgesic-overuse headache evolving from episodic migraine. Brain 129(Pt 2):543-550

15. Bechara A, Damasio AR, Damasio H, Anderson SW (1994) Insensitivity to future consequences following damage to human prefrontal Cortex. Cognition 50:7-15

16. Silberstein SD, Olesen J, Bousser MG et al (2005) The International Classification of Headache Disorders, 2nd edition (ICHDII) - revision of criteria for 8.2 Medication-overuse headache. Cephalalgia 25:460-465

17. Stewart WF et al (2001) Development and testing of the Migraine Disability Assessment (MIDAS) Questionnaire to assess headache-related disability. Neurology 56(6 Suppl 1):S20-S28

18. D'Amico D et al (2001) The Migraine Disability Assessment (MIDAS) questionnaire: translation and reliability of the Italian version. Cephalalgia 21(10):947-952 
19. Gossop M et al (1997) Test-retest reliability of the Severity of Dependence Scale. Addiction 92(3):353

20. Grande RB et al (2009) The Severity of Dependence Scale detects people with medication overuse: the Akershus study of chronic headache. J Neurol Neurosurg Psychiatry 80(7):784-789
21. Grazzi L et al (2001) Chronic migraine with medication overuse pre-post withdrawal of symptomatic medication: clinical results and fMRI correlations. Headache 50(6):998-1004 